\title{
Strain rate effect in material testing of bulk adhesive
}

\author{
W. $\operatorname{Pan}^{1} \&$ R. Schmidt ${ }^{2}$ \\ ${ }^{I}$ Research and Technology, DaimlerChrysler AG, Stuttgart, Germany \\ ${ }^{2}$ Institute of General Mechanics, RWTH Aachen University, Germany
}

\begin{abstract}
Due to its high energy absorption and flexibility in joining different materials, adhesive bonding is applied increasingly in automobile and aircraft structures that may be subjected to high loading rates and large dynamic loads encountered in a crash or impact. Simulation of the dynamic response of bonded structures needs reliable numerical models accounting for the strain rate effects. Unlike that of steel, there is still no standardized testing procedure for adhesive materials designed for crash or impact loading conditions. Hence, how to obtain and transfer the dynamic test results to numerical models is an important issue for simulation. Besides using empirical formulae for material models, alternatively strain rate effects are frequently taken into account by direct implementation of experimentally determined data as material input curves, e.g. in finite element codes like LS-DYNA etc. Each of those curves consists of a plot of stress vs. strain within constant strain rate, but due to the acceleration of the specimen and its yielding within the gauge length, mostly the strain rate is not constant. Different kinds of strain rate e.g. nominal, averaged, actual strain rate etc. are discussed in this paper. The actual strain rate increases during the entire high-speed loading process of the test specimen. Frequently the plots of strain rate vs. time exhibit strong oscillations. Often even the designated loading velocities are not reached by far. Thus using experimentally determined stress-strain curves for the designated loading velocity without any consideration of the real strain rate evolution during the experiment may lead to insufficient accuracy. In this paper, a conversion method is suggested for interpreting such stress-strain curves despite the increasing strain rate during high-speed loading experiments and to obtain the corresponding stress for explicit algorithms used in transient analyses.
\end{abstract}

Keywords: strain rate, high-speed loading, adhesives, crash simulation. 


\section{Introduction}

Study of adhesively bonded joints under static loading conditions has a long history concerning both theory and experiment. A review of the literature shows considerable efforts to obtain reliable estimations through numerical simulation (see e.g. [1-5]). However, concerning the dynamic behaviour under high-speed loading conditions the simulation results are still not satisfactory. Since the adhesive layers are very thin, it is very difficult to implement them into finite element models including a suitable material model. Above all, the transferring of dynamic bulk testing results plays an important role for the material modelling. This concerns especially the experimentally observed strain rate effect of adhesives in uniaxial tensile testing.

In [6] a detailed investigation of the evolution of strains in metallic high-speed tensile test specimens revealed that the strain rate is not constant during the experiment, but increases nonlinearly with time. Moreover, yielding occurs before the nominal strain rate has been reached. Therefore, it was suggested to determine the strain rate at $0.2 \%$ plastic strain and to use this value, e.g. in the Cowper-Symonds function, instead of the nominal strain rate associated with the designated loading velocity.

Normally the material data provided in suppliers' datasheets are obtained from tensile, lap shear, T-shear and impact peel tests. However, these data are not directly applicable for FE computation and also inadequate to develop suitable material models for the dynamic behaviour of adhesively bonded joints.

Except for ideal-plastic material and engineering applications for which the strain rate effect can be ignored, the consideration of a large range of strain rates is required in crash or impact problems. In the present paper results of high-speed tensile tests of bulk adhesive are investigated in detail. A method is suggested on how to use the experimental results despite of the increasing strain rate during the test, and how to transfer them to transient analyses using explicit algorithms.

\section{Strain rate in uniaxial tensile tests}

To characterise the mechanical properties of adhesive material, three basic tests are generally required, i.e. tension, compression and shear test. Till now those tests are mostly conducted in static or quasi-static experiments. For dynamic applications, like e.g. crash and impact loading, there are still no standardized test procedures, to obtain reliable experimental data and to transfer them to numerical modelling and simulation. Some related standards can be referred to ISO or DIN, e.g.:

- DIN ISO EN 8256: Plastics - Determination of tensile impact strength

- DIN ISO EN 604: Plastics - Determination of compressive properties

- DIN EN 14869-2: Structural adhesives - Determination of shear behaviour of structural bonds, Part 2. Thick adherents shear test 
Fig. 1 and table 1 show a specimen for uniaxial tensile tests according to DIN ISO EN 8256.

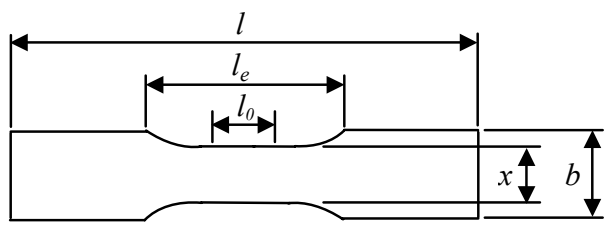

Figure 1: $\quad$ Specimen geometry.

Table 1: $\quad$ Specimen dimensions.

\begin{tabular}{|c|c|c|c|c|c|c|}
\hline Type & $l$ & $b$ & $x$ & $l_{0}$ & $l_{e}$ & $r$ \\
\hline 3 & $80 \pm 2$ & $15 \pm 0.2$ & $10 \pm 0.2$ & $10 \pm 0.2$ & $30 \pm 2$ & $20 \pm 1$ \\
\hline
\end{tabular}

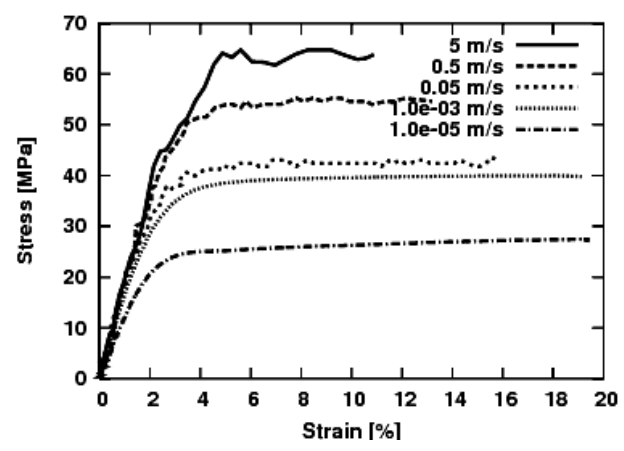

Figure 2: $\quad$ Stress-strain curves for EP 208.

Fig. 2 shows results of tensile tests reported in [8] for EP 208 conducted under 5 loading velocities: $5 \mathrm{~m} / \mathrm{s}, 0.5 \mathrm{~m} / \mathrm{s}, 0.05 \mathrm{~m} / \mathrm{s}, 1.0 \mathrm{e}-03 \mathrm{~m} / \mathrm{s}$ and $1.0 \mathrm{e}-05$ $\mathrm{m} / \mathrm{s}$. Obviously, the plots of the engineering stress versus engineering strain show a behaviour that is typical for viscoelastic materials. One can observe an increasing stiffness as the loading velocity increases. Later the curves bend at different and indistinct "yield stresses" and reach large strains $(10 \sim 20 \%)$ at failure.

Normally, the strain rate is calculated by

$$
\dot{\varepsilon}=\frac{V_{0}}{l_{0}}
$$

where $l_{0}$ is the gauge length and $V_{0}$ denotes the loading velocity. Hence, for the specimen in fig. 1 with a gauge length of $0.01 \mathrm{~m}$, one obtains a strain rate of $5001 / \mathrm{s}$ for the loading velocity $5 \mathrm{~m} / \mathrm{s}$. 
Such a value can be called "nominal strain rate". However, the use of $l_{0}$ in the above calculation is questionable, which is demonstrated in the following for the case of plastic deformation. In this case the determination of the strain rate by eq. (1) is only correct, if yielding occurs within the gauge length of $10 \mathrm{~mm}$. However, in fig. 3 it can be seen that the plastic (whitened) zone is not exclusively concentrated in the gauge length of $10 \mathrm{~mm}$, but extends to the fixed length of $30 \mathrm{~mm}$.

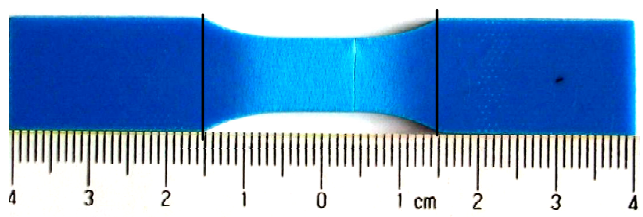

Figure 3: $\quad$ Tested tensile specimen.

Thus the strain rate can be rather assumed to be

$$
\dot{\varepsilon}=\frac{5 \mathrm{~m} / \mathrm{s}}{0.03 \mathrm{~m}}=167 \mathrm{~s}^{-1} \text {. }
$$

In fact, even for constant nominal loading velocity the strain rate changes all the time during the loading process. This can be seen in fig. 4a from the plot of the strain rate vs. time for the uniaxial tensile test of EP 208 in fig. 2. In fig. 4a the strain rate in a time interval between $t_{j}<t<t_{i}$ has been calculated as

$$
\dot{\varepsilon}=\frac{\Delta \varepsilon}{\Delta t}=\frac{\varepsilon_{i}-\varepsilon_{j}}{t_{i}-t_{j}} .
$$

These averaged values also depend strongly on the chosen time interval because of the oscillating test result.

Thus the overall averaged strain rate is

$$
\overline{\dot{\varepsilon}}=\frac{\sum_{i=1}^{n} \dot{\varepsilon}_{i}}{n} .
$$

From fig. $4 \mathrm{~b}$ it can be concluded that the loading velocity is not constant but increases during the entire process. The curves of the displacement vs. time in fig. $4 \mathrm{~b}$ can be well approximated by second order polynomial equations indicated in these graphs (see also table 2). That means that the specimens are always in the process of acceleration during the testing.

According to the total loading time $T$, using the polynomial expressions obtained in fig. $4 \mathrm{~b}$, the final reached velocities can be calculated as given in table 2.

Apparently, the designated loading velocities $V_{o}$ were not reached even till the moment the specimens failed.

With the final velocities reached in the test, the associated maximum strain rates can be calculated as 


$$
\dot{\varepsilon}_{\max }=\frac{\left.V(t)\right|_{t=T}}{l_{0}} .
$$

The four kinds of strain rates discussed above in eqs. (1), (2), (4) and (5) are listed in table 3 .
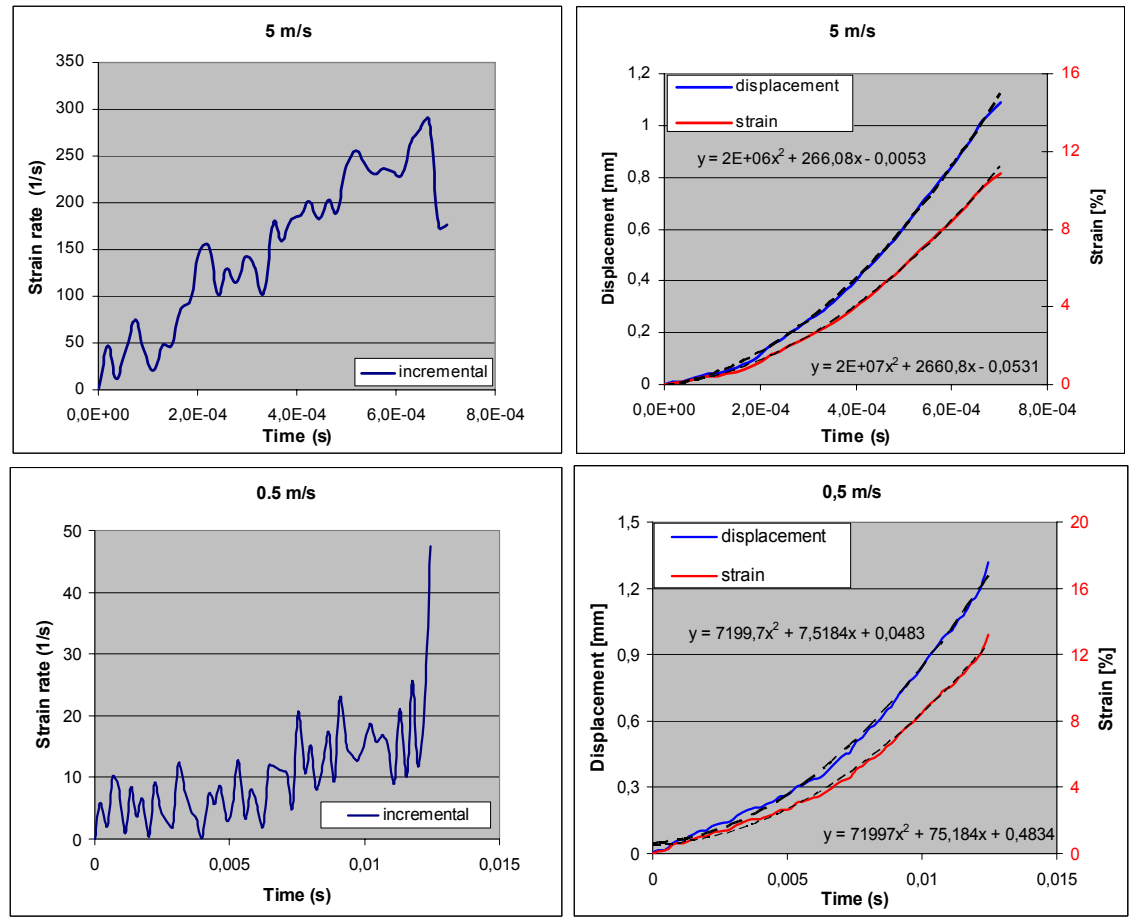

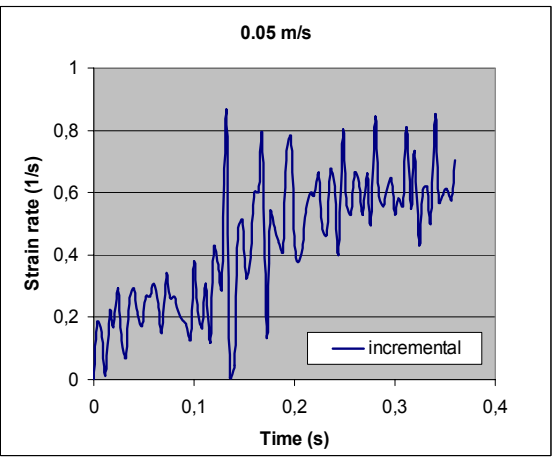

(a)

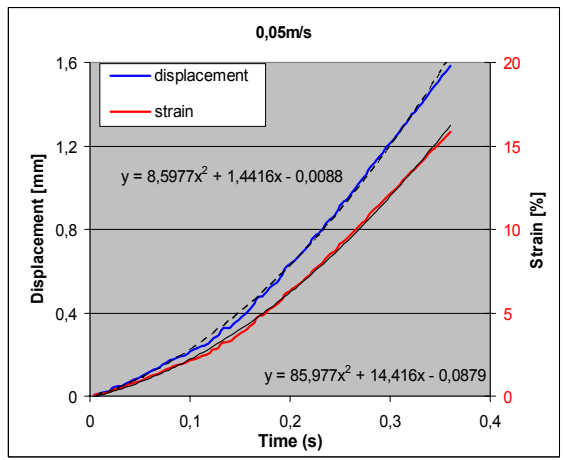

(b)

Figure 4: (a): Strain rate vs. time for 3 impact velocities (left); (b): Displacement and strain vs. time for 3 impact velocities (right). 
Table 2: $\quad$ Final velocities reached in the test.

\begin{tabular}{|r|c|c|c|}
\hline $\mathbf{V}_{\mathbf{0}}[\mathbf{m} / \mathbf{s}]$ & Displacement $[\mathbf{m m}]$ vs. Time[s] & Total Time[s] & V $_{\text {reached }}$ \\
\hline 5 & $S=2 \mathrm{e}+06 t^{2}+266.08 t-0.0053$ & $7.04 \mathrm{e}-04$ & $3.08 \mathrm{~m} / \mathrm{s}$ \\
\hline 0.5 & $S=7199.7 t^{2}+7.5184 t+0.0483$ & $1.24 \mathrm{e}-02$ & $1.86-01 \mathrm{~m} / \mathrm{s}$ \\
\hline 0.05 & $S=8.5977 t^{2}+1.4416 t-0.0088$ & 0.36 & $7.63 \mathrm{e}-03 \mathrm{~m} / \mathrm{s}$ \\
\hline
\end{tabular}

Table 3: $\quad$ Loading velocities vs. strain rates.

\begin{tabular}{|c|c|c|c|c|c|}
\hline \multirow{2}{*}{$\begin{array}{c}\text { Strain rate } \\
\left(\mathrm{s}^{-1}\right)\end{array}$} & \multicolumn{5}{|c|}{ Designated loading velocities $(\mathrm{m} / \mathrm{s})$} \\
\cline { 2 - 6 } & 5 & 0.5 & 0.05 & $1.0 \mathrm{e}-03$ & $1.0 \mathrm{e}-05$ \\
\hline Nominal & 500 & 50 & 5 & 0.1 & $1.0 \mathrm{e}-03$ \\
\hline Assumed & 167 & 16.7 & 1.67 & $3.0 \mathrm{e}-04$ & $3.0 \mathrm{e}-06$ \\
\hline Averaged & 155 & 10.6 & 0.44 & 0.035 & $7.5 \mathrm{e}-04$ \\
\hline Maximum & 308 & 18.6 & 0.763 & $\mathrm{n} / \mathrm{a}$ & $\mathrm{n} / \mathrm{a}$ \\
\hline
\end{tabular}

\section{Material input curves for simulation}

Besides using empirical formulae for material models, experimentally determined data are also directly implemented as material input curves in many finite element programs, like e.g. LS-DYNA.

Fig. 5 shows a group of such material input curves. Each curve is listed for a certain strain rate. This requires that each plot of stress vs. strain is determined for a certain constant strain rate. Typically, this constant strain rate of each material input curve is assumed to be the nominal strain rate or the averaged strain rate from the experiment.

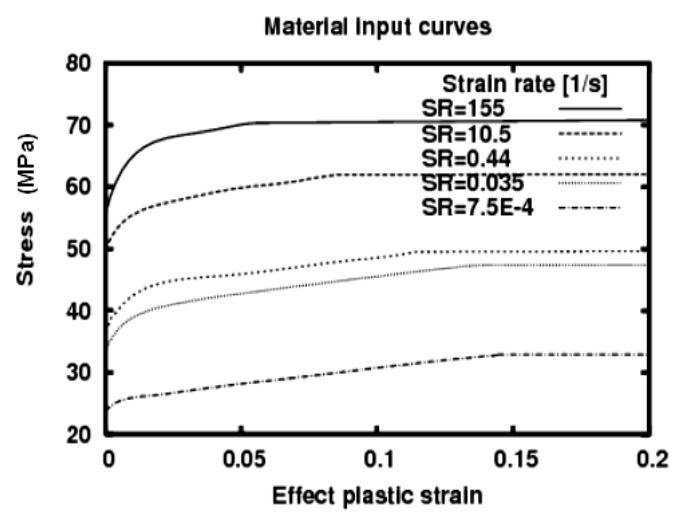

Figure 5: $\quad$ Example of material input curves for LS-DYNA.

During transient analysis in each time step the current strain rate is calculated in order to determine, which of the material input curves has to be chosen. 
Normally this implies interpolation between neighbouring curves. Then the current stress can be determined according to the current strain and strain rate.

In what follows, we shall take into account that in experimentally determined plots of stress vs. strain the strain rate is not constant but increases during the entire process. Table 4 shows the range of the strain rates that actually occur during the tensile tests in fig. 2 . The values of the strain rate in the right column have been calculated by differentiating the polynomial expressions for the strain evolution in fig. $4 \mathrm{~b}$ with respect to time and inserting the total loading time $T$ given in table 2 . One can observe e.g., that for the designated loading velocity $5 \mathrm{~m} / \mathrm{s}$ the strain rate ranges from $26.60 \sim 308.091 / \mathrm{s}$.

Table 4: $\quad$ Strain rate range for 3 loading velocities.

\begin{tabular}{|c|c|}
\hline $\begin{array}{c}\text { Loading Velocity } \\
{[\mathbf{m} / \mathbf{s}]}\end{array}$ & $\begin{array}{c}\text { Strain rate } \\
{[\mathbf{1} / \mathbf{s}]}\end{array}$ \\
\hline 5 & $26.60 \sim 308.09$ \\
\hline 0.5 & $0.75 \sim 18.67$ \\
\hline 0.05 & $0.14 \sim 0.76$ \\
\hline
\end{tabular}

According to fig. $4 \mathrm{~b}$ for the experimental tensile tests to be discussed here, the strain evolution can be approximated by a polynomial function of time in the form $\varepsilon(t)=a t^{2}+b t+c$. Accordingly, the strain rate is $\dot{\varepsilon}(t)=2 a t+b$. Thus the relation of strain rate vs. strain is obtained as

$$
\varepsilon=\frac{1}{4 a}\left(\dot{\varepsilon}^{2}+4 a c-b^{2}\right) .
$$

With the values of $a, b$, and $c$ given in table 2, one obtains e.g. for $V_{0}=5 \mathrm{~m} / \mathrm{s}$ the relation $\dot{\varepsilon}^{2}=8 e+3 \varepsilon+11.33 e+2$, which has been plotted in fig. 6 .

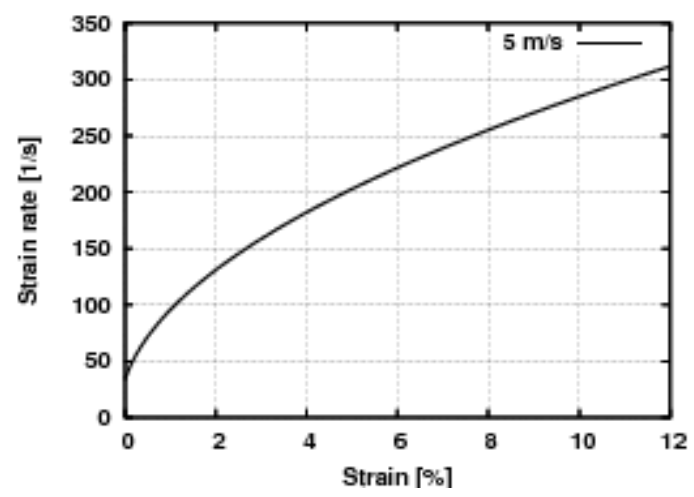

Figure 6: Strain rate vs. strain for $V_{0}=5 \mathrm{~m} / \mathrm{s}$. 
The difference between material input curves for FE-simulation (solid lines) and an experimental curve (dashed line) can be schematically illustrated by the 3D plot in fig. 7, where now additionally the information concerning the strain rate (see fig. 6) has been included. The experimentally obtained curve of stress vs. strain exhibits a range of strain rates from $\dot{\varepsilon}_{i}$ to $\dot{\varepsilon}_{j}$. Note that the plot of strain rate vs. strain in fig. 6 can be found in the horizontal plane of fig. 7 as the projection of the experimental curve.

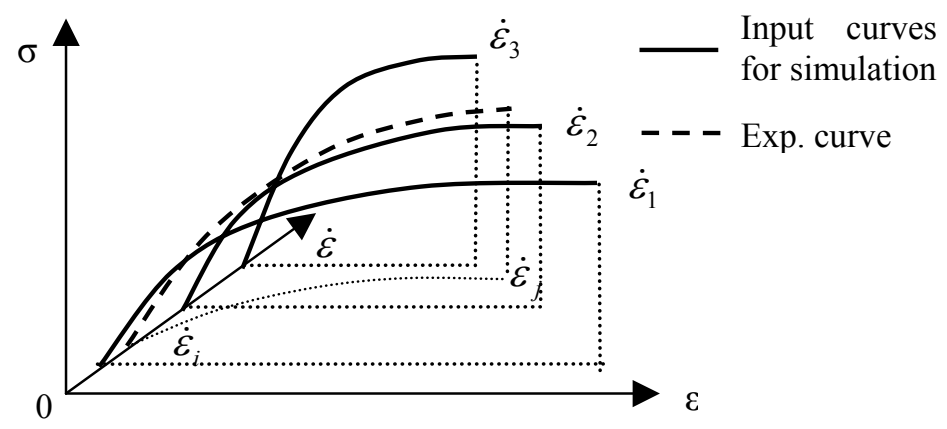

Figure 7: Plots of the experimentally obtained results and of material input curves for numerical simulations.

\section{Interpolation method}

Taking the above considerations into account, it follows that for strain rate dependent materials not only the input matrix of stress vs. strain is needed for material input curves. Additionally, a third column consisting of the strain rate associated with the respective strain has to be included into the input matrix.

Because of the oscillations occurring in the dynamic test, the incremental strain rate (see eq. (3) and fig. 4a) cannot be implemented readily. However, an approximation function of the strain rate vs. time, $\dot{\varepsilon}(t)$, can be derived from the plot of strain vs. time (see fig. 4b). Thus each material input curve consists of 3 columns of strain, strain rate and stress for each loading velocity used in the experimental tests, e.g.

$$
\left[\begin{array}{ccc}
\varepsilon_{1} & \dot{\varepsilon}_{1} & \sigma_{1} \\
\varepsilon_{2} & \dot{\varepsilon}_{2} & \sigma_{2} \\
\varepsilon_{3} & \dot{\varepsilon}_{3} & \sigma_{3} \\
\cdots & \cdots & \cdots
\end{array}\right]_{V_{0}=5 \mathrm{~m} / \mathrm{s}}
$$


In explicit FE computations, the 3-column matrix of material input curves can be used as follows, with $(i, j, k)$ referring to the experimental curve IDs, $(0,1,2)$ referring to points on the curves, and 0 denoting the seeking point:

1. For each time step the displacements are to be calculated, and simultaneously the current strains and strain rates.

2. Since the current strain $\varepsilon_{0}$ normally is not one of the values of the first column of the above input matrix, interpolation has to be performed between two adjacent values (e.g. $\varepsilon_{i 1}, \varepsilon_{i 2}$ ) of each experimental curve $i$.

3. Next, for each strain $\varepsilon_{i 0}=\varepsilon_{0}$, the corresponding strain rates $\dot{\varepsilon}_{i 0}$ have to be interpolated in the same way.

4. From the three strain rates a 2 nd-order polynomial equation can be derived in the form: $\sigma=f(\dot{\varepsilon})=\alpha \dot{\varepsilon}^{2}+\beta \dot{\varepsilon}+\gamma$. Thus, the corresponding stress can be obtained according to the current strain and strain rate.

Fig. 8 shows the 3-D contour built using the experimentally obtained curves for three different loading velocities $(5 \mathrm{~m} / \mathrm{s}, 0.5 \mathrm{~m} / \mathrm{s}, 0.05 \mathrm{~m} / \mathrm{s})$. A cutting plane of current strain rate $\dot{\varepsilon}_{0}$ is constructed with three dashed lines. Hence the corresponding stress value can be interpolated from those of the 3 material input curves.

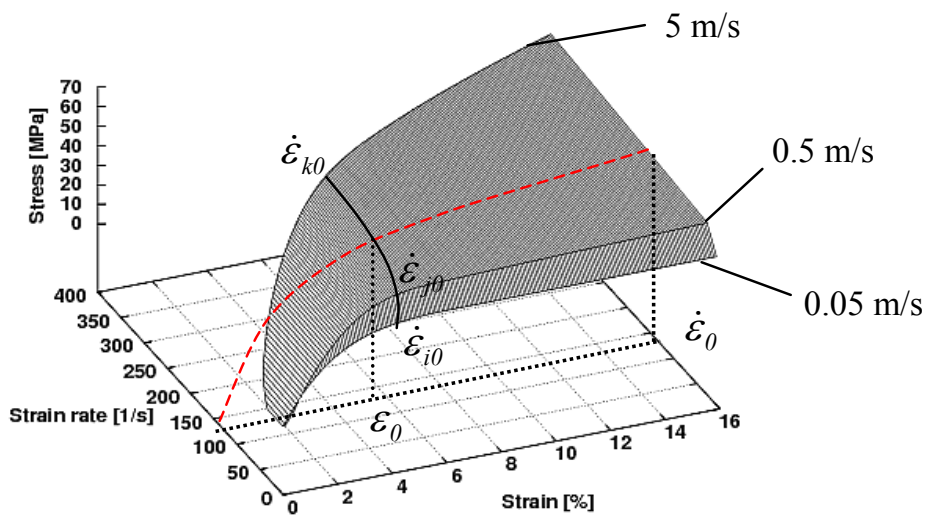

Figure 8: $\quad 3-D$ contour built using three experimentally obtained curves.

\section{Conclusion}

In this paper the basic tests for adhesive material concerning high-speed application are discussed. Experimental results of uniaxial tensile tests of bulk adhesive at various loading velocities are investigated in detail. It is shown that 
due to the high dynamic effects the strain rates are not constant during high-speed tensile tests. Consequently, the paper deals with the question, how experimentally determined stress-strain curves obtained for various nominal loading velocities can be used for material modelling despite of the fact that the strain rates increase with time during these tests. The proposed method is based on the construction of a 3-D contour using the experimentally determined stress-strain curves. The change of strain rates during the tests is calculated from the plots of strain vs. time and subsequently taken into account by plotting the experimental results in the 3-D space of stress vs. strain and strain rate. Finally, an interpolation method is outlined to determine the stresses from the available experimental data.

\section{Acknowledgements}

The authors gratefully acknowledge Dr. T. Disse, DaimlerChrysler AG, Germany, for the experimental data.

\section{References}

[1] Reddy, J.N. \& Roy, S., Nonlinear analysis of adhesively bonded joints, Int. J. Non-Linear Mech., 23, pp. 97-112, 1988.

[2] Reddy, J.N. \& Roy, S., Finite-Element analysis of adhesive joints, Adhesive Bonding, ed. Liang-Huang Lee, 359-393, Plenum Press, New York 1991.

[3] Krasucki, F., Münch, A. \& Ousset, Y., Numerical simulation of debonding of adhesively bonded joint, Int. J. Solids and Structures, 39, pp. 6355-6383, 2002.

[4] Abdel Wahab, M.M., Ashcroft, I.A. \& Crocombe, A.D., A comparison of failure prediction methods for an adhesively bonded composite beam, $J$. Strain Analysis, 39, pp. 173-185, 2004.

[5] Hahn, O., Untersuchung zum Festigkeitsverhalten von Metallklebung bei Schlagbeanspruchung, Dissertation, RWTH Aachen, 1972.

[6] Hahn, O., Kurzok, J.R., Oeter, M., Brede, M., Hesebeck, O., Dilger, K. \& Schmid, G., Untersuchungen zum Crashverhalten geklebter und hybridgefügter Stahlblechverbindungen, Stahl-Zentrum, Studiengesellschaft Stahlanwendung e.V., Report 477, Verlag und Vertriebsgesellschaft mbH, Düsseldorf, 2002.

[7] Disse, T., Untersuchungen zum geschwindigkeitsabhängigen Festigkeitsund Verformungsverhalten von Klebverbindungen, Dissertation, Shaker Verlag Aachen, 2004. 